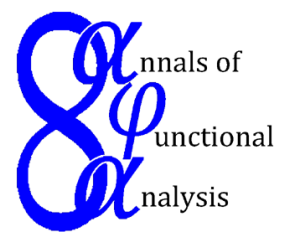

Ann. Funct. Anal. 7 (2016), no. 1, 96-101
http://dx.doi.org/10.1215/20088752-3334778
ISSN: 2008-8752 (electronic)
http://projecteuclid.org/afa

\title{
INJECTION THEOREM FOR LOCAL DITKIN SETS
}

\author{
ANTOINE DERIGHETTI \\ Dedicated to Professor Anthony To-Ming Lau \\ Communicated by K. F. Taylor
}

\begin{abstract}
For Figà-Talamanca-Herz algebras $A_{p}(G), 1<p<\infty$, of a locally compact group $G$ and a closed subgroup $H$ of $G$, we prove an injection theorem for local Ditkin sets.
\end{abstract}

\section{INTRODUCTION AND PRELIMINARIES}

Let $G$ be a locally compact abelian group, let $H$ be a closed subgroup, and let $F$ be a closed subset of $H$. The following result is well known and classical (see [8, Theorem 7.4.13]): $F$ is a Ditkin set in $G$ if and only if $F$ is a Ditkin set in the subgroup $H$. In this article we extend this statement to a class of noncommutative groups, including the amenable groups (Theorem 4.1). In this generalization we replace $L^{1}(\widehat{G})$ by the Fourier algebra $A_{2}(G)$ or, more generally, by the Figà-Talamanca-Herz algebra $A_{p}(G)$. Partial results (for $p=2$ or for every $1<p<\infty)$ are already known. For normal subgroups see [2, Théorème 12], and for neutral subgroups see [1, Corollary 7$])$. For other directly related works, see also E. Kaniuth and A. Lau's [5, Theorem 3.4] and K. Parthasarathy and N. S. Kumar's [7, Theorem 3.5].

We use a natural action (denoted $u \cdot T$ ) of $A_{p}(G)$ on the Banach space $\mathcal{L}$ of all bounded operators of $L^{p}(G)$. Our proof requires a new characterization of the notion of locally $p$-Ditkin sets involving not only convolution operators but also general bounded operators of $L^{p}$. A closed subset $F$ of $G$ is locally p-Ditkin if and

Copyright 2016 by the Tusi Mathematical Research Group.

Received Apr. 23, 2015; Accepted May 11, 2015.

2010 Mathematics Subject Classification. Primary 43A15; Secondary 43A46, 43A22.

Keywords. abstract harmonic analysis, locally compact group, $L^{p}$-spaces and other function spaces on groups, special sets on groups. 


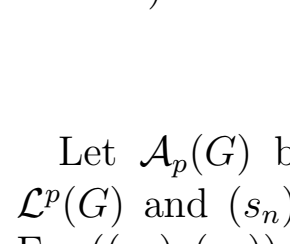

Ann. Funct. Anal. 7 (2016), no. 1, 96-101
http://dx.doi.org/10.1215/20088752-3334778
ISSN: 2008-8752 (electronic)
http://projecteuclid.org/afa

\title{
INJECTION THEOREM FOR LOCAL DITKIN SETS
}

\author{
ANTOINE DERIGHETTI \\ Dedicated to Professor Anthony To-Ming Lau \\ Communicated by K. F. Taylor
}

\begin{abstract}
For Figà-Talamanca-Herz algebras $A_{p}(G), 1<p<\infty$, of a locally compact group $G$ and a closed subgroup $H$ of $G$, we prove an injection theorem for local Ditkin sets.
\end{abstract}

\section{INTRODUCTION AND PRELIMINARIES}

Let $G$ be a locally compact abelian group, let $H$ be a closed subgroup, and let $F$ be a closed subset of $H$. The following result is well known and classical (see [8, Theorem 7.4.13]): $F$ is a Ditkin set in $G$ if and only if $F$ is a Ditkin set in the subgroup $H$. In this article we extend this statement to a class of noncommutative groups, including the amenable groups (Theorem 4.1). In this generalization we replace $L^{1}(\widehat{G})$ by the Fourier algebra $A_{2}(G)$ or, more generally, by the Figà-Talamanca-Herz algebra $A_{p}(G)$. Partial results (for $p=2$ or for every $1<p<\infty)$ are already known. For normal subgroups see [2, Théorème 12], and for neutral subgroups see [1, Corollary 7$])$. For other directly related works, see also E. Kaniuth and A. Lau's [5, Theorem 3.4] and K. Parthasarathy and N. S. Kumar's [7, Theorem 3.5].

We use a natural action (denoted $u \cdot T$ ) of $A_{p}(G)$ on the Banach space $\mathcal{L}$ of all bounded operators of $L^{p}(G)$. Our proof requires a new characterization of the notion of locally $p$-Ditkin sets involving not only convolution operators but also general bounded operators of $L^{p}$. A closed subset $F$ of $G$ is locally p-Ditkin if and

Copyright 2016 by the Tusi Mathematical Research Group.

Received Apr. 23, 2015; Accepted May 11, 2015.

2010 Mathematics Subject Classification. Primary 43A15; Secondary 43A46, 43A22.

Keywords. abstract harmonic analysis, locally compact group, $L^{p}$-spaces and other function spaces on groups, special sets on groups. 


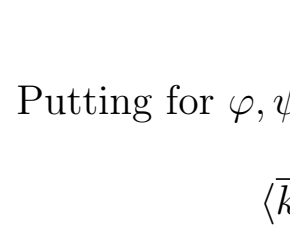

Ann. Funct. Anal. 7 (2016), no. 1, 96-101
http://dx.doi.org/10.1215/20088752-3334778
ISSN: 2008-8752 (electronic)
http://projecteuclid.org/afa

\title{
INJECTION THEOREM FOR LOCAL DITKIN SETS
}

\author{
ANTOINE DERIGHETTI \\ Dedicated to Professor Anthony To-Ming Lau \\ Communicated by K. F. Taylor
}

\begin{abstract}
For Figà-Talamanca-Herz algebras $A_{p}(G), 1<p<\infty$, of a locally compact group $G$ and a closed subgroup $H$ of $G$, we prove an injection theorem for local Ditkin sets.
\end{abstract}

\section{INTRODUCTION AND PRELIMINARIES}

Let $G$ be a locally compact abelian group, let $H$ be a closed subgroup, and let $F$ be a closed subset of $H$. The following result is well known and classical (see [8, Theorem 7.4.13]): $F$ is a Ditkin set in $G$ if and only if $F$ is a Ditkin set in the subgroup $H$. In this article we extend this statement to a class of noncommutative groups, including the amenable groups (Theorem 4.1). In this generalization we replace $L^{1}(\widehat{G})$ by the Fourier algebra $A_{2}(G)$ or, more generally, by the Figà-Talamanca-Herz algebra $A_{p}(G)$. Partial results (for $p=2$ or for every $1<p<\infty)$ are already known. For normal subgroups see [2, Théorème 12], and for neutral subgroups see [1, Corollary 7$])$. For other directly related works, see also E. Kaniuth and A. Lau's [5, Theorem 3.4] and K. Parthasarathy and N. S. Kumar's [7, Theorem 3.5].

We use a natural action (denoted $u \cdot T$ ) of $A_{p}(G)$ on the Banach space $\mathcal{L}$ of all bounded operators of $L^{p}(G)$. Our proof requires a new characterization of the notion of locally $p$-Ditkin sets involving not only convolution operators but also general bounded operators of $L^{p}$. A closed subset $F$ of $G$ is locally p-Ditkin if and

Copyright 2016 by the Tusi Mathematical Research Group.

Received Apr. 23, 2015; Accepted May 11, 2015.

2010 Mathematics Subject Classification. Primary 43A15; Secondary 43A46, 43A22.

Keywords. abstract harmonic analysis, locally compact group, $L^{p}$-spaces and other function spaces on groups, special sets on groups. 


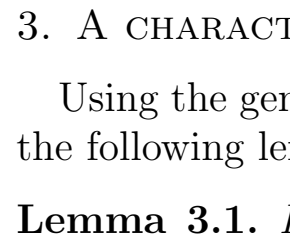

Ann. Funct. Anal. 7 (2016), no. 1, 96-101
http://dx.doi.org/10.1215/20088752-3334778
ISSN: 2008-8752 (electronic)
http://projecteuclid.org/afa

\title{
INJECTION THEOREM FOR LOCAL DITKIN SETS
}

\author{
ANTOINE DERIGHETTI \\ Dedicated to Professor Anthony To-Ming Lau \\ Communicated by K. F. Taylor
}

\begin{abstract}
For Figà-Talamanca-Herz algebras $A_{p}(G), 1<p<\infty$, of a locally compact group $G$ and a closed subgroup $H$ of $G$, we prove an injection theorem for local Ditkin sets.
\end{abstract}

\section{INTRODUCTION AND PRELIMINARIES}

Let $G$ be a locally compact abelian group, let $H$ be a closed subgroup, and let $F$ be a closed subset of $H$. The following result is well known and classical (see [8, Theorem 7.4.13]): $F$ is a Ditkin set in $G$ if and only if $F$ is a Ditkin set in the subgroup $H$. In this article we extend this statement to a class of noncommutative groups, including the amenable groups (Theorem 4.1). In this generalization we replace $L^{1}(\widehat{G})$ by the Fourier algebra $A_{2}(G)$ or, more generally, by the Figà-Talamanca-Herz algebra $A_{p}(G)$. Partial results (for $p=2$ or for every $1<p<\infty)$ are already known. For normal subgroups see [2, Théorème 12], and for neutral subgroups see [1, Corollary 7$])$. For other directly related works, see also E. Kaniuth and A. Lau's [5, Theorem 3.4] and K. Parthasarathy and N. S. Kumar's [7, Theorem 3.5].

We use a natural action (denoted $u \cdot T$ ) of $A_{p}(G)$ on the Banach space $\mathcal{L}$ of all bounded operators of $L^{p}(G)$. Our proof requires a new characterization of the notion of locally $p$-Ditkin sets involving not only convolution operators but also general bounded operators of $L^{p}$. A closed subset $F$ of $G$ is locally p-Ditkin if and

Copyright 2016 by the Tusi Mathematical Research Group.

Received Apr. 23, 2015; Accepted May 11, 2015.

2010 Mathematics Subject Classification. Primary 43A15; Secondary 43A46, 43A22.

Keywords. abstract harmonic analysis, locally compact group, $L^{p}$-spaces and other function spaces on groups, special sets on groups. 


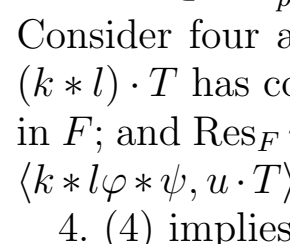

Ann. Funct. Anal. 7 (2016), no. 1, 96-101
http://dx.doi.org/10.1215/20088752-3334778
ISSN: 2008-8752 (electronic)
http://projecteuclid.org/afa

\title{
INJECTION THEOREM FOR LOCAL DITKIN SETS
}

\author{
ANTOINE DERIGHETTI \\ Dedicated to Professor Anthony To-Ming Lau \\ Communicated by K. F. Taylor
}

\begin{abstract}
For Figà-Talamanca-Herz algebras $A_{p}(G), 1<p<\infty$, of a locally compact group $G$ and a closed subgroup $H$ of $G$, we prove an injection theorem for local Ditkin sets.
\end{abstract}

\section{INTRODUCTION AND PRELIMINARIES}

Let $G$ be a locally compact abelian group, let $H$ be a closed subgroup, and let $F$ be a closed subset of $H$. The following result is well known and classical (see [8, Theorem 7.4.13]): $F$ is a Ditkin set in $G$ if and only if $F$ is a Ditkin set in the subgroup $H$. In this article we extend this statement to a class of noncommutative groups, including the amenable groups (Theorem 4.1). In this generalization we replace $L^{1}(\widehat{G})$ by the Fourier algebra $A_{2}(G)$ or, more generally, by the Figà-Talamanca-Herz algebra $A_{p}(G)$. Partial results (for $p=2$ or for every $1<p<\infty)$ are already known. For normal subgroups see [2, Théorème 12], and for neutral subgroups see [1, Corollary 7$])$. For other directly related works, see also E. Kaniuth and A. Lau's [5, Theorem 3.4] and K. Parthasarathy and N. S. Kumar's [7, Theorem 3.5].

We use a natural action (denoted $u \cdot T$ ) of $A_{p}(G)$ on the Banach space $\mathcal{L}$ of all bounded operators of $L^{p}(G)$. Our proof requires a new characterization of the notion of locally $p$-Ditkin sets involving not only convolution operators but also general bounded operators of $L^{p}$. A closed subset $F$ of $G$ is locally p-Ditkin if and

Copyright 2016 by the Tusi Mathematical Research Group.

Received Apr. 23, 2015; Accepted May 11, 2015.

2010 Mathematics Subject Classification. Primary 43A15; Secondary 43A46, 43A22.

Keywords. abstract harmonic analysis, locally compact group, $L^{p}$-spaces and other function spaces on groups, special sets on groups. 


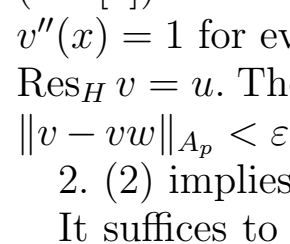

Ann. Funct. Anal. 7 (2016), no. 1, 96-101
http://dx.doi.org/10.1215/20088752-3334778
ISSN: 2008-8752 (electronic)
http://projecteuclid.org/afa

\title{
INJECTION THEOREM FOR LOCAL DITKIN SETS
}

\author{
ANTOINE DERIGHETTI \\ Dedicated to Professor Anthony To-Ming Lau \\ Communicated by K. F. Taylor
}

\begin{abstract}
For Figà-Talamanca-Herz algebras $A_{p}(G), 1<p<\infty$, of a locally compact group $G$ and a closed subgroup $H$ of $G$, we prove an injection theorem for local Ditkin sets.
\end{abstract}

\section{INTRODUCTION AND PRELIMINARIES}

Let $G$ be a locally compact abelian group, let $H$ be a closed subgroup, and let $F$ be a closed subset of $H$. The following result is well known and classical (see [8, Theorem 7.4.13]): $F$ is a Ditkin set in $G$ if and only if $F$ is a Ditkin set in the subgroup $H$. In this article we extend this statement to a class of noncommutative groups, including the amenable groups (Theorem 4.1). In this generalization we replace $L^{1}(\widehat{G})$ by the Fourier algebra $A_{2}(G)$ or, more generally, by the Figà-Talamanca-Herz algebra $A_{p}(G)$. Partial results (for $p=2$ or for every $1<p<\infty)$ are already known. For normal subgroups see [2, Théorème 12], and for neutral subgroups see [1, Corollary 7$])$. For other directly related works, see also E. Kaniuth and A. Lau's [5, Theorem 3.4] and K. Parthasarathy and N. S. Kumar's [7, Theorem 3.5].

We use a natural action (denoted $u \cdot T$ ) of $A_{p}(G)$ on the Banach space $\mathcal{L}$ of all bounded operators of $L^{p}(G)$. Our proof requires a new characterization of the notion of locally $p$-Ditkin sets involving not only convolution operators but also general bounded operators of $L^{p}$. A closed subset $F$ of $G$ is locally p-Ditkin if and

Copyright 2016 by the Tusi Mathematical Research Group.

Received Apr. 23, 2015; Accepted May 11, 2015.

2010 Mathematics Subject Classification. Primary 43A15; Secondary 43A46, 43A22.

Keywords. abstract harmonic analysis, locally compact group, $L^{p}$-spaces and other function spaces on groups, special sets on groups. 